\title{
Understanding the Quadrilateral Concept of Junior High School Students Based on APOS Theory in Terms of Differences in Cognitive Styles
}

\author{
A.C. Anam \\ Postgraduate Mathematics Education \\ Program \\ Universitas Negeri Surabaya \\ Indonesia \\ ahmad.17070785015@mhs.unesa.ac.id
}

\author{
D. Juniati \\ Department of Mathematics \\ Universitas Negeri Surabaya \\ Indonesia \\ dwijuniati@unesa.ac.id
}

\author{
P. Wijayanti \\ Department of Mathematics \\ Universitas Negeri Surabaya \\ Indonesia \\ pradnyowijayanti@unesa.ac.id
}

\begin{abstract}
This study was designed to describe the understanding of quadrilateral concepts in junior high school students based on the APOS theory (Action, Process, Objects, and Schemes). The research used qualitative approach to explain the understanding of two junior high school students who had equal mathematical abilities and each had fielddependent (FD) and field-independent (FI) cognitive style with descriptive explanations. Quadrilateral task and interviews guidelines were used to explore student's quadrilateral concept. The results of this study indicated that for the action stage, both could solve understanding problems related to quadrilateral concept. For the process stage, the FI subject explained the process or the calculation coherently in every step taken in problem with understanding the characteristic of shapes to define quadrilateral concept, meanwhile the FD subject explained the process with interpreting quadrilateral concepts and calculating all steps to find the shapes area in problem verbally. In the object stage, both could compare two or more shapes in quadrilateral problem and prove steps to find areal of shapes correctly in problem. For the scheme stage, FI subject could infer the relationship between shape's properties in quadrilateral to explain quadrilateral concepts and make conclusions. In the other hand, the FD subject did not have abilities in schema stage like FI subject performed.
\end{abstract}

Keywords-Understanding, Quadrilateral concept, APOS theory, cognitive style

\section{INTRODUCTION}

Understanding is the result of positive connections in relating new experiences received with experiences previously owned [1]. Understanding is important key that students should have in creating cognitive relationships during the process of learning activities. This is in accordance with one of the learning objectives in mathematics listed in 2013 curriculum: understanding of mathematical concepts, explaining the relevance of concepts, and applying concepts or algorithms in flexibly, accurately, efficiently, and precisely to solve the problems. The understanding of math concepts means to make the relationship between mental representation of someone with mathematical conception being studied [2]. Mathematics concepts are not only a single idea but also an idea in the schematic structure of schematic knowledge, thus it requires complex abilities and thoroughness to master the concept [3]. In general, understanding a concept in an individual cannot be observed precisely, but it can be determined with several approaches through indicators used to observe the understanding of the concept.

In mathematics, the mathematical concepts require maximum mastery to understand them. One of mathematics concepts that must be understood is geometry concept, because we cannot separate from shapes of geometry [4]. Quadrilateral is a geometry shape that we often found, "quadrilateral is the union of four-line segment joined in the four coplanar points and had four sides [5]". In other hand, several research results indicated that students understanding of geometry were generally weak, thus they still needed certain treatment to increase the understanding of geometry with new learning $[4,6]$. Better level of thinking and cognitive skills in students can be acquired if mathematical concepts are understood comprehensively, such as the understanding of geometry concept. Students' understanding of mathematical concepts will help improve their cognitive skills if they can process the concepts actively studied, thus students will be able to represent every concept according to the level of understanding they have as well as to incorporate it into a structure of mathematical knowledge [3]. Processing the learned concept requires mental activity developed in every stage using reflective abstraction in Piaget's knowledge theory. APOS theory is a construction theory of student's understanding related to a concept through the stages of action, processes, objects, and schemes. The theory was developed by Dubinsky prior adopted from Piaget's theory of reflective abstraction which stated that there is a tendency of each individual in responding and understanding mathematical problems by using reflective abstraction in a social context by reconstructing actions, processes, objects and schemas in mathematical concepts and applying them in 
a scheme to secure a problem and solve mathematical problems [7].

APOS theory also helps to understand the mechanism of a reflective abstraction to describe the development of an individual's logical thinking, and its expansion of ideas in several mathematical concepts [8]. APOS is an understanding using physical and mental activity transforming objects obtained from the results of interactions with other people or the results of mental construction with pre-existing objects related to mathematical ideas [3]. Mental construction is conducted through several stages: action, process, object, and schema. Action is a type of understanding of mathematical concepts involving mental or physical transformation due to reactions to external stimulation based on concepts learned as the previous explanations presented. Process is defined as a type of understanding of mathematical concept involving imagination in transforming mental objects as controlled internal activities. In other words, during process stage, students can find out the concepts without external activities or external stimuli. Object means the stages of understanding mathematical concepts constructed from the application of activities at the stages of action and process, thus students can understand a mathematical concept with the help of external stimulation, then transform it into internal and controlled activities, and finally they can understand the concepts and meaning of the concept well. The last stage is schema, which involves a process of understanding concepts to link activities of action, processes, and objects. It also involves the construction of other schemas. Students who carry out the stages of the schema understand a concept and the relationship of a concept with any concepts in other topics. During stages on APOS to construct individual understanding, differences commonly occurred; generally due to cognitive style because it refers to the way a person thinks and processes information exactly to understand a concept. $[9,10,11]$

\section{METHOD}

This research was a qualitative research with descriptive approach. The purpose of this research was to describe the understanding of students' concepts in quadrilateral material based on the APOS theory systematically, practically, and comprehensively. The subject of the research was taken to conduct several activities, such as forming a group of students in the class at junior high schools in Surabaya. There were 28 students determined in the class (4 males and 24 females). They were given a mathematical ability test and GEFT test. The aim of mathematical ability test was to determine the students' different abilities in mathematical test, while GEFT test was to show their respective cognitive style. Based on the tests, the reseacher found two students with the same gender, mathematical ability and cognitive style as the research subjects. Then, the research subjects were given a quadrilateral task to describe the quadrilateral students' concept.

The instrument used in this research was a mathematical ability test that contained questions adapted from the national examination. There were 10 questions adapted from multiple choices given to the 28 students, while GEFT test was taken from Witkin to determine field-independent (FI) and field dependent (FD) cognitive style [11]. There were 18 items in GEFT test used to classify the cognitive style; students answering 18 items in 0-9 intervals correct answer was students with FD cognitive style, while students answered 18 items in 10-18 intervals correct answer was students with FI cognitive style. Then, quadrilateral task contained four questions in quadrilateral material adjusted to APOS understanding indicators was given with aim to determine students' understanding of quadrilateral concepts. The quadrilateral tasks given to number 1 and 2 were used to identify and analyze the understanding of the subject. At the action stage, number 3 was used to find out and analyze the subject's understanding. At the process stage, number 4 was used to identify and analyze the subject's understanding at the stage object. The last item had more complex criteria than practice items because it contained applicative questions related to other concept used to analyze the subject's understanding at the stage of the scheme. Interview used to increase data information from the research subject not found on the quadrilateral task.

Data analysis on quadrilateral task was based on the results of the students' answer towards the problem. It was used to describe the understanding of quadrilateral concepts of junior high school students based on APOS theory. Table 1 below presents several indicators based on APOS theory.

TABLE I. CRITERIA FOR STUDENT UNDERSTANDING INDICATORS BASED ON APOS THEORY IN QUADRILATERAL MATERIAL

\section{Understanding indicators}

Action Determine the characteristics of quadrilateral with calculation of area of quadrilateral shapes to found quadrilateral concept

Process Explain how to determine the characteristic of a quadrilateral and quadrilateral concepts

Object Make comparison between quadrilateral shapes to explains quadrilateral concepts when only one of the shapes elements is known

Schema Make a connection between quadrilateral shapes to find conclusion and infer other relationships to other shapes or material in similar topics

\section{RESULT AND DISCUSSIONS}

This research used mathematical ability test, GEFT test, and quadrilateral task. The result of mathematical ability test showed 28 students who had taken the test could be grouped into three categories: three students $(10 \%)$ have high mathematical skills, ten students $(35 \%)$ have medium mathematical skills, and fifteen students $(55 \%)$ have low mathematical skills. GEFT test resulted that from 28 students, two groups with ten students $(35 \%)$ had FI cognitive style, and 18 students $(65 \%)$ had FD cognitive style. Based on the results of the data analysis, the researcher found and selected two students with same gender, medium level of mathematical ability skills, and each subject had respectively FI and FD cognitive style. Details of the results from tests given is presented on Table 2 . 
TABLE II. THE RESULT OF GEFT AND MATH ABILITY TEST

\begin{tabular}{cccc}
\hline & Gender & $\begin{array}{c}\text { Math ability } \\
\text { skill }\end{array}$ & Cognitive style \\
\cline { 2 - 4 } $\begin{array}{c}\text { Subject } 1 \\
\text { (AIA) } \\
\begin{array}{c}\text { Subject 2 } \\
\text { (BS) }\end{array}\end{array}$ & Male & Medium & Field-independent \\
\hline
\end{tabular}

The following description contains explanation of research result on students' understanding of concept with quadrilateral task given to two students as research subject and the result of interview with detailed explanation.

\section{A. An overview of the research result based on subject 1}

Quadrilateral task consisted of five questions showed that the subject had finished all task well. Detailed explanation about understanding quadrilateral concepts based on APOS was presented in the following description.

a) Action stage. Quadrilateral task on number one and number two consisted of the problem based on APOS theory in action stage which showed that subject could determine elements and characteristics of each shapes in quadrilateral and also discovered the concept of each of shapes based on other characteristic. In addition, subject calculated the circumference of one of the shapes in quadrilateral correctly, even though there was mistake in calculating the circumference. Thus, subject had understood and carried out all steps in action stage.

b) Process stage. Number three item on quadrilateral task indicated a problem which showed process stage on APOS theory. The results of tasks, subject informed the other characteristic of shapes shown in the problem. The subject also explained the concept of each shape based on these characteristics through his answer in calculating the area of shapes. Furthermore, the results of the interview showed that subject could explain coherently every step taken in problem with understanding the characteristic of shapes to define quadrilateral concept. Subject solved in steps when calculating the area of shapes and his answer was correct. Hence, he completed the process stage.

c) Object stage. Comparation between shapes in quadrilateral was the goal of item number four. Subject could compare two similar shapes with different sizes. Subject found the area of shapes in quadrilateral and other with known one element based on the following analysis and calculation: given circumference of square $=16 \mathrm{~cm}$, sides $A$ $(S A)=16: 4=4 \mathrm{~cm}$. We knew $s=4 \mathrm{~cm}$. to answer square areas of $\mathrm{A}$, thus:

$$
\begin{aligned}
& \mathrm{L} A=s^{2} \square A=\mathrm{s}^{2} \\
& =4^{2}=16 \mathrm{~cm}^{2}=4^{2}=16 \mathrm{~cm}^{2}, \\
& \text { because LB }=3 \times \text { LA then, }
\end{aligned}
$$

$\mathrm{L} \square B=3 \times 16=48 \mathrm{~cm}^{2}$

$$
B=3 \times 16=48 \mathrm{~cm}^{2}
$$

Thus, area of the other shape is $48 \mathrm{~cm}^{2}$

Furthermore, the researcher conducted an interview to clarify the answers that he was finished. Interview with subject proved that he had understood the problem of tasks and performed in steps according to indicators in object stage.

d) Schema stage. At this stage, subject was expected to be able to combine all the previous stages (action, process, and object) by making a relationship each other. Subject connected properties in quadrilateral shapes and found the concept based on the results of these connections. He also made conclusions that shape of question number five was a combination trapezoid and square. The following was the results of the process:

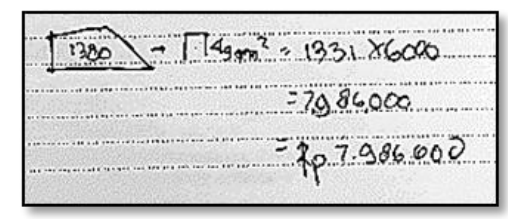

Fig. 1. Task result of subject

The results of task above showed that subject answered correctly. He calculated area of shape by adding two areas asked, that they were trapezoid area and square area. Subject understood the problem as well as made a connection of quadrilateral material with others because it had understood problem when interviewed. Besides, he also clarified steps to compute quadrilateral shape area in this question. Thus, he had carried out the final stage in APOS theory, which was schema.

\section{B. An overview of the research result based on subject 2}

Subject could explain the problem of quadrilateral concept fluently, although there are some errors that found in the result of task and confirmation through with interviews. Detailed explanation of understanding the quadrilateral concept by subject based on APOS theory is shown below:

a) Action stage. Subject could determine the characteristic of shape in quadrilateral to find the concept shown in items number one and two. In item number one, which contained the command to determine the characteristic of quadrilateral shape, he could determine all of the shape's properties well. However, in question number two that consisted of the command to calculate the circumference of shape, he had wrong answer. Results analyzed by researcher confirmed that the subject related to the answer through interviews. The results showed that subject explained the concept of quadrilateral by determining the characteristics shown before. He could also calculate the circumference of shape, but there was an error in count. Thus, subject could perform all phases of the action stage well even though there were some not in appropriation.

b) Process stage. At the process stage indicated by item number three, subject could explain the concept of shape in quadrilateral well based on other properties (other than mentioned in action stage). In addition, he also calculated the shape area even though it did not show detailed steps in the 
results of task. At the time of interview, the subject was able to explain these concepts and represented all steps in calculating the area shown in quadrilateral verbally. Thus, phases in the process stage had been passed well.

c) Object stage. The results of subject in item number four showed that subject was able to compare between shapes in quadrilateral, especially square shapes where only one of the elements was known and then computed their area. First, subject wrote shapes area A with a circumference $16 \mathrm{~cm}$. Given that there were 4 sides in square, then subject found 1616

out a length of its sides with formula 44 that result was 4 . Formula of square shape is $s^{2}$, then he wrote it directly with $\frac{16}{4} \times \frac{16}{4}=16 \mathrm{~cm}^{2} \frac{16}{4} \times \frac{16}{4}=16 \mathrm{~cm}^{2}$ to search square area B with multiplies shape A $x 3$ that had result of $48 \mathrm{~cm}^{2}$. Based on the results of this task, subject compared each of shapes with their elements and he understood it. When interviewed, subject described each of steps in this problem well and coherently. Thus, he had passed through all steps in the object stage.

d) Schema stage. The last stage in APOS theory was schema. It was related to the indicators on item number 5. The results of task on this item showed that subject did not understand the problem well and there was an error. This was because he had not been able to make the relationship between shapes in quadrilateral and not able to connect each stage in APOS theory properly. Thus, subject had not fully implemented the stages of the schema properly.

\section{CONCLUSIONS}

All things considered, it could be inferred that subject who had field-independent and field-dependent cognitive style showed different answers in working on the problem that given. The results of this study indicated that for the action stage, both could solve problems related to quadrilateral concept. For the process stage, the FI subject explained the process or the calculation coherently in every step taken in problem with understanding the characteristic of shapes to define quadrilateral concept, meanwhile the FD subject explained the process with interpreting quadrilateral concepts and calculating all steps to find the shapes area in problem verbally. The object stage, both could compare between two or more shapes in quadrilateral in problem, and could prove steps to find shapes area correctly in problem. For the schema stage, The FI subject could make a relationship with connected between shape's properties in quadrilateral to was explain quadrilateral concepts with made conclusions based on the result of these connections. Otherwise, the FD subject could not perform abilities in schema stage just like FI subject done. Thus, there were differences between FI and FD subject in understanding of quadrilateral concept based on APOS theory.

\section{REFERENCES}

[1] A. Ilana, et al., "APOS Theory: A Framework for Research and Curriculum Development in Mathematics Education," New York: Springer, 2014.

[2] P. Barmby, T. Harries, S. Higgins, and J. Suggate, "How can We Assess Mathematical Understanding," Proceedings on the $31^{\text {st }}$ of the International Group for the Psychology of Mathematics Education PME Seoul, vol. 2 pp. 41-48, 2007.

[3] Weyer and R. Sarah, "APOS Theory as a Conceptualization for Understanding Mathematical Learning," Summation - Mathematics and Computer Science Scholarship at Ripon Collage, pp. 9-15, 2010.

[4] M.M.L. Puloo, D. Juniati, and P. Wijayanti, "Visualization Profile of Junior High School Students in Solving Geometry Problems Viewed from Gender Differences," J. Phy : Conf. Series vol.1108, ID 012063 , 2018.

[5] A.A. Safitri, D. Juniati, and Masriyah, "Students' Relational Understanding in Quadrilateral Problem Solving Based on Adversity Quotient,”J. Phy : Conf. Series, vol. 947, ID 012039, 2017.

[6] P.K. Agus, A.C. Anam, Abdussakir, and R. Imam, "Integrasi Etnomatematika dengan Model Pembelajaran Probing-prompting untuk melatih Komunikasi Matematis Siswa," Mapan: Jurnal Matematika dan Pembelajaran, vol. 7, pp. 1-15, 2019.

[7] D. Haylock and A. Cockburn, "Understanding Mathematics for Young Children, United Kingdom: SAGE Publication, 2008.

[8] S. Bansilal, B. Deonarain, and T. Maria, "An APOS Study on PreService Teachers' Understanding of Injections and Surjections," J. Maths, Beh., vol. 48, pp. 22-37, 2017.

[9] Altun, Arif, and M. Cakan, “Undergraduate Students' Academic Achievement, Field Independent/Dependent Cognitive Styles and Attitude Toward Computers," Edu. Tech. Soc., vol. 9, pp. 289-297, 2006.

[10] A.J. Barody, Y. Feil, and A.R. Johson, "An Alternative Reconceptualization of Procedural and Conceptual Knowledge," J. Res. Maths. Edu., vol 38. pp. 115-131, 2007.

[11] H.A. Witkin and R.G. Donald, "Cognitive Styles: Essence and Origins,” New York: International Universities Press, 1981. 